\title{
IMPACTO DO EXERCÍCIO CONTÍNUO E INTERVALADO NA RESPOSTA AUTONÔMICA E PRESSÓRICA EM 24 HORAS
}

\author{
IMPACT OF CONTINUOUS AND INTERVAL EXERCISE ON THE AUTONOMIC AND PRESSORIC \\ RESPONSE IN 24 HOURS
}

Artigo Original

Original ARTICLE

Artículo Original

\author{
IMPACTO DEL EJERCICIO CONTINUO Y INTERVALICO SOBRE LA RESPUESTA AUTONÓMICA Y \\ PRESORA EN 24 HORAS
}

\author{
Juliano Casonatto ${ }^{1}$ (Educador Físico) \\ Veridiana Domingues' \\ (Educadora Física) \\ Diego Giulliano Destro Christofaro² \\ (Educador Físico) \\ 1. Universidade Norte do Paraná \\ (UNOPAR), Educação Física, \\ Londrina, Paraná, Brasil. \\ 2. Universidade Estadual Paulista \\ (UNESP), Educação Física, \\ Presidente Prudente, São Paulo, \\ Brasil.
}

\section{Correspondência:}

Universidade Norte do Paraná, Centro de Ciências Biológicas e da Saúde. Rua Marselha, 591, Jardim Piza, Londrina, PR. 86041-120. juliano2608@hotmail.com

\section{RESUMO}

Introdução: Exercícios físicos aeróbicos contínuos são os mais relacionados com a hipotensão pós-exercício. No entanto, são escassas as informações sobre o impacto do exercício intervalado sobre a resposta pressórica de 24 horas. Objetivo: Comparar as respostas pressóricas subagudas e agudas em uma sessão de exercício aeróbico contínuo e intervalado e identificar possíveis modulações em indicadores de atividade autonômica em adultos normotensos. Métodos: Submeteram-se 25 adultos normotensos saudáveis a três sessões experimentais: controle (30 minutos em repouso), exercício contínuo (30 min. - 60\%-70\% da FC res) e exercício intervalado (6 sessões de 5 minutos com intervalos de 2 min. - 60-70\% da $\mathrm{FC}_{\text {res }}$ ) e seus parâmetros cardiovasculares foram monitorados por 24 horas após as sessões. Para comparação dos dados, foi utilizada a ANOVA para medidas repetidas, seguida de suas hipóteses. Resultados: No acompanhamento subagudo foi identificada redução significativa $(P<0,05)$ da pressão arterial sistólica somente após a sessão de exercício contínuo em comparação com a sessão de repouso $(115 \pm 2 \mathrm{mmHg}$ vs. $112 \pm 2 \mathrm{mmHg}$ ) e à sessão controle $(119 \pm 2 \mathrm{mmHg}$ vs. $112 \pm 2 \mathrm{mmHg})$. Não se identificou redução da pressão arterial ambulatorial em nenhuma das sessões experimentais. Os indicadores autonômicos parassimpáticos (RMSSD e pNN50) permaneceram reduzidos após 30 minutos em ambas as sessões de exercício. Conclusão: Uma única sessão de exercício aeróbico contínuo causa redução subaguda da pressão arterial em adultos normotensos. A sessão única de exercício aeróbico contínuo e intervalado não promove redução da pressão arterial ambulatorial na média nos períodos intermediários de sono e vigília.

Descritores: hipotensão pós-exercício; monitorização ambulatorial; pressão arterial.

\section{ABSTRACT}

Introduction: Continuous aerobic exercises are the most related to post-exercise hypotension. However, there is little information on the impact of interval exercises on blood pressure response in 24 hours. Objective: To compare subacute and acute pressure responses of a continuous and interval aerobic exercise session and identify possible modulations in autonomic activity indicators in normotensive adults. Methods: Twenty-five healthy normotensive adults underwent three experimental sessions: control (30 minutes at rest), continuous exercise (30 min, 60-70\% HRres) and interval exercise (six 5-minute sessions with 2 min intervals - 60-70\% HRres), and their cardiovascular parameters were monitored for 24 hours after sessions. To compare the data, we used ANOVA for repeated measures, followed by their hypotheses. Results: Significant reduction was identified in the subacute follow-up $(P<0.05)$ in systolic blood pressure only after continuous exercise session, compared to the rest session $(115 \pm 2 \mathrm{mmHg}$ vs. $112 \pm 2 \mathrm{mmHg}$ ) and control (119 $\pm 2 \mathrm{mmHg}$ vs. $112 \pm 2 \mathrm{mmHg}$ ) sessions. No ambulatory blood pressure reduction was found in any of the experimental sessions. The parasympathetic autonomic indicators (RMSSD and pNN50) remained reduced after 30 minutes in both exercise sessions. Conclusion: A single continuous aerobic exercise session causes subacute reduction in blood pressure in normotensive adults. A single session of continuous and interval aerobic exercise does not promote reduction of ambulatory blood pressure in interim periods of sleep and wakefulness.

Keywords: post-exercise hypotension; monitoring, ambulatory; arterial pressure.

\section{RESUMEN}

Introducción: Los ejercicios aeróbicos continuos son los más relacionados con la hipotensión post-ejercicio. No obstante, existe poca información sobre el impacto del ejercicio intervalico en la respuesta presora durante 24 horas. Objetivo: Comparar las respuestas presoras subagudas y agudas en una sesión de ejercicio aeróbico continuo e intervalico e identificar posibles modulaciones de los indicadores de la actividad autonómica en adultos normotensos. Métodos: Se sometieron 25 adultos sanos normotensos a tres sesiones experimentales: control (30 minutos en reposo), ejercicio continuo (30 min. - 60\%-70\% de la FCres) y ejercicio intervalico (6 sesiones de 5 minutos con intervalos de 2 min. -60\%-70\% de la F(res) y sus parámetros cardiovasculares fueron monitorizados durante 24 horas después de las sesiones. Para comparar los datos, se utilizó ANOVA para medidas repetidas, seguida de sus hipótesis. Resultados: En el seguimiento subagudo se identificó una reducción significativa $(P<0,05)$ en la presión arterial sistólica solamente después de la sesión de ejercicio continuo en comparación con la sesión de reposo $(115 \pm 2 \mathrm{mmHg}$ versus $112 \pm 2$ $\mathrm{mm} \mathrm{Hg}$ ) y la sesión de control (119 $2 \mathrm{mmHg}$ versus $112 \pm 2 \mathrm{mmHg}$ ). No se ha verificado la reducción de la presión arterial ambulatoria en ninguna de las sesiones experimentales. Los indicadores autonómicos parasimpáticos (RMSSD 
y pNN50) permanecieron reducidos después de 30 minutos en ambas sesiones de ejercicio. Conclusión: Una sola sesión de ejercicios aeróbicos continuos causa reducción subaguda de la presión arterial en adultos normotensos. Una sola sesión de ejercicio aeróbico continuo e intervalico no promueve ninguna reducción de la presión arterial ambulatoria en los períodos intermedios de sueño y vigilia.

Descriptores: hipotensión posejercicio; monitoreo ambulatorio; presión arterial.

\section{INTRODUÇÃO}

A hipotensão pós-exercício se caracteriza pela redução da pressão arterial de repouso nos minutos ou horas subsequentes à realização de uma sessão de exercício em relação aos valores pré-exercício ${ }^{1}$. 0 interesse em relação à hipotensão pós-exercício tem sido motivado principalmente em função das suas implicações clínicas, uma vez que pode ser considerada um agente não farmacológico auxiliar, no tratamento e prevenção de disfunções relacionadas ao sistema cardiovascular, como a hipertensão arterial sistêmica².

Nesse sentido, para que a hipotensão pós-exercício tenha importância clínica, é preciso que o efeito hipotensivo e perdure pelo maior tempo possível com magnitude de queda significativa. Dessa forma, diversos estudos buscaram investigar os efeitos ambulatoriais de uma única sessão de exercício aeróbico ${ }^{3-5}$ e resistido ${ }^{6-9}$ sobre a pressão arterial. Ressalta-se que ambos tipos de exercício promovem hipotensão pós-exercício, no entanto, o exercício aeróbico parece ser mais eficiente ${ }^{6}$.

As sessões de exercício aeróbico têm se caracterizado por atividades físicas de intensidade contínua que perduram em média 40 minutos, porém essas atividades não são bem suportadas por indivíduos acometidos por limitações funcionais, como problemas osteo-articulares. Nesse caso, os exercícios do tipo intervalado, caracterizado por atividades intermitentes com curtos períodos de ação e recuperação, têm sido propostos como alternativa ao modelo clássico de exercício contínuo, inclusive para redução crônica da pressão arterial ${ }^{10}$.

Por outro lado, informações sobre as respostas pressóricas agudas após a realização de exercício aeróbico intervalado são raras. Além disso, não foram identificadas informações em relação à hipotensão pós-exercício intervalado obtidas por meio da monitorização ambulatorial de 24 horas em indivíduos normotensos. Dessa forma, exercícios intervalados podem se configurar como alternativa aos exercícios contínuos no que se refere aos impactos positivos na pressão arterial.

Assim, o objetivo do presente estudo foi comparar as respostas pressóricas subagudas e agudas de uma sessão de exercício aeróbico contínuo e intervalado e identificar possíveis modulações em indicadores de atuação autonômica em adultos normotensos.

\section{MÉTODOS}

O cálculo do tamanho da amostra para teste de hipótese para uma média (desvio-padrão $=10 \mathrm{mmHg}^{11}$; diferença a ser detectada $=5 \mathrm{mmHg}^{11}$; significância=5\%; poder estatístico=80\%) indicou a necessidade de 25 sujeitos. Nesse sentido, foram recrutados 28 voluntários de ambos os sexos (10 masculino e 18 feminino), todos adultos (>18 anos), normotensos não praticantes de atividade física regular e sistematizada e sem comprometimentos osteomusculares que prejudicasse a prática de exercício físico. Todos os sujeitos não faziam uso de fármacos de ação adrenérgica. Além disso, como critérios iniciais de inclusão, os sujeitos não poderiam ser fumantes e nem fazer o uso de álcool e/ ou bebidas cafeinadas por pelo menos 12 horas antes das sessões de exercício, além de estarem ausentes da prática de exercício físico e/ ou atividade física vigorosa por pelo menos 24 horas.
Após serem informados sobre os procedimentos do estudo, todos os indivíduos assinaram termo de consentimento livre e esclarecido, de acordo com as normas da Resolução 196/96 do Conselho Nacional de Saúde. Este estudo foi aprovado pelo Comitê de Ética em Pesquisa da Universidade Norte do Paraná (Plataforma Brasil, CAAE no: 10682912.6.0000.0108).

Foram determinadas as medidas de massa corporal por meio de uma balança antropométrica digital (Urano, OS 180A, Canoas, Brasil), graduada de 0 a 150 kg, com precisão de 0,1kg; e estatura com a utilização de um estadiômetro de madeira, com escala de precisão de 0,1 cm, de acordo com os procedimentos descritos por Gordon et al. ${ }^{12}$. O índice de massa corporal (IMC) da amostra foi calculado pelo quociente massa corporal $\div$ estatura ${ }^{2}$, sendo a massa corporal expressa em quilogramas $(\mathrm{kg})$ e a estatura em metros (m).

A pressão arterial de repouso foi mensurada utilizando um monitor automático (Omron MX3 Plus, Bannockburn, EUA) previamente validado para medida clínica da pressão arterial em adultos ${ }^{13}$. Inicialmente, os sujeitos permaneceram 20 minutos confortavelmente sentados em ambiente calmo, ameno e livre de ruídos. Após os primeiros 10 minutos, a pressão arterial foi mensurada três vezes em intervalos de 5 minutos, ou seja, as medidas foram realizadas aos 10 minutos, 15 minutos e 20 minutos. A média das três aferições foi considerada como a pressão arterial de repouso. As aferições foram realizadas de acordo com as recomendações da Associação Americana do Coração ${ }^{14}$.

A variabilidade da frequência cardíaca foi monitorada juntamente com a pressão arterial, por meio de um monitor de frequência cardíaca (Polar RS800CX, Kempele, Finlândia) previamente validado ${ }^{15}$. Os intervalos R-R foram gravados no equipamento transferidos para um computador por meio do software Polar Pro Trainer 5 (Kempele, Finlândia). A transformação de Fourier foi utilizada para quantificar as bandas de baixa $\left(L F_{R-R}{ }^{n u}\right)$ e alta frequência $\left(H_{R-R}{ }^{n u}\right)$ em unidades normalizadas, de acordo com as recomendações da Força Tarefa da Sociedade Europeia de Cardiologia e da Sociedade Norte-Americana de Eletrofisiologia ${ }^{16}$. Além disso, a análise no domínio do tempo foi obtida pelos índices SDNN (desvio padrão da média de todos os intervalos RR normais); RMSSD (raiz quadrada da média das diferenças sucessivas ao quadrado, entre os intervalos R-R normais adjacentes); pNN50 (percentagem de intervalos RR normais adjacentes com diferença de duração maior que 50 milissegundos). A análise das variáveis relacionadas ao domínio do tempo e da frequência foi realizada em janelas de cinco (repouso pré-exercício) e 30 (repouso pós-exercício) minutos, com a utilização do software Kubios HRV versão 2.2 (Kuopio, Finlândia).

A monitorização ambulatorial da pressão arterial foi realizada por meio de um equipamento oscilométrico automático (Dyna-MAPA) acoplado no braço não dominante, seguindo os procedimentos descritos pela Associação Americana do Coração ${ }^{14}$. Os sujeitos foram orientados a manterem o braço imóvel durante as medidas. O monitor foi calibrado por comparação direta, realizada por um observador previamente treinado por meio de um esfigmomanômetro de mercúrio, seguindo as recomendações da Associação Americana do Coração ${ }^{14}$. O monitor foi programado para registrar a pressão arterial sistólica, diastólica e a frequência cardíaca a cada 20 minutos, com exceção do período compreendido entre às $23 \mathrm{~h} 00$ e 08h00, o qual foi registrado a cada 30 
minutos, afim de minimizar distúrbios no sono. O painel do equipamento foi ocultado para impedir feedback dos indivíduos. Foi solicitado aos sujeitos que registrassem o horário de vigília e sono que eram repassados no dia seguinte, durante o procedimento de retirada do equipamento. O registro médio de medidas válidas ficou acima de $90 \%$.

A percepção subjetiva de esforço foi avaliada por meio da escala padronizada de Borg (6-20 pontos) ${ }^{17}$ a cada 5 minutos, durante a realização da sessão de exercício aeróbico contínuo e intervalado. No caso da sessão de exercício intervalado, os sujeitos eram solicitados a reportar a percepção subjetiva esforço imediatamente ao momento de finalização de cada série de 5 minutos.

Antes do início do experimento, os sujeitos foram submetidos às avaliações antropométricas e hemodinâmicas em repouso. Na sequência, foi calculada a"zona alvo" de treinamento (60-70\% da FC res) para cada indivíduo, baseada na previsão da frequência cardíaca máxima (FCmax=220 - idade) para estabelecer a intensidade do exercício.

Em um segundo momento, os sujeitos foram encaminhados a um ambiente calmo, ameno e livre de ruídos, onde as medidas de pressão arterial de repouso foram mensuradas durante um período de 20 minutos em posição sentada. Em seguida, os sujeitos foram submetidos à execução da sessão de exercício em esteira ergométrica, sendo contínuo (SEC), intervalado (SEI), onde foi dado um intervalo passivo de dois minutos a cada cinco minutos de exercício, nesse intervalo foi reduzida a velocidade até a parada total da esteira e sessão controle (SC) na qual os sujeitos permaneceram sentados por 30 minutos. Os dados resumidos em relação às configurações de exercícios são apresentados na Tabela 1. Vale destacar que em momento prévio foi realizada a aleatorização dos sujeitos nas sessões de exercício, com a utilização de uma Tabela de números aleatórios, gerada especificamente para esse fim (http://www.random.org/). Além disso, foi realizado um período de aquecimento prévio com duração de 5 minutos, no qual a intensidade foi progressivamente aumentada até que se atingisse a intensidade de execução (60-70 da $\mathrm{FC}_{\mathrm{res}}$ ). Após o término do esforço, a velocidade foi progressivamente reduzida até a parada total do sujeito, com duração também de 5 minutos. As sessões experimentais foram realizadas em ordem contrabalanceada separadas por um período mínimo de 48 horas.

Após a realização das sessões de exercício, os sujeitos foram submetidos à fase de análise laboratorial pós-exercício (60 min), para o acompanhamento das variáveis cardiovasculares (pressão arterial e variabilidade da frequência cardíaca). Para tanto, os sujeitos permaneceram sentados em ambiente calmo, ameno e livre de ruídos. Após esse período foi dado um intervalo ( 15 min) para o sujeito tomar banho e trocar de roupa antes de ser instalado o equipamento para verificação ambulatorial da pressão arterial por 24 horas. No dia seguinte às sessões experimentais os sujeitos retornavam ao laboratório para a retirada do equipamento.

\section{Tratamento estatístico}

O teste $t$ independente foi aplicado para comparação entre os sexos, precedido da verificação da homogeneidade dos dados, por meio do teste de Levene's. Em caso de violação da homogeneidade (massa corporal), foi aplicada a correção recomendada. Em um segundo momento, os dados foram submetidos ao teste de Mauchly's para verificação da

Tabela 1. Características gerais da amostra.

\begin{tabular}{c|c|c|c|c|c}
\hline & \multicolumn{2}{|c|}{ Masculino $(\mathbf{n}=10)$} & \multicolumn{2}{c|}{ Feminino $(\mathbf{n}=15)$} & \multirow{2}{*}{$\boldsymbol{P}$} \\
\cline { 2 - 5 } & Média & DP & Média & DP & \\
\hline Idade (anos) & 32,10 & 7,78 & 31,47 & 10,91 & 0,876 \\
\hline Massa Corporal $(\mathrm{kg})$ & 69,87 & 6,20 & 68,03 & 12,38 & 0,628 \\
\hline Estatura $(\mathrm{m})$ & 1,65 & 0,09 & 1,68 & 0,07 & 0,344 \\
\hline IMC $\left(\mathrm{kg} / \mathrm{m}^{2}\right)$ & 25,70 & 3,35 & 23,85 & 3,27 & 0,184 \\
\hline $\mathrm{DP}=$ desvio padrão; $\mid \mathrm{MC}=$ indice de massa corporal.
\end{tabular}

esfericidade. Em nenhuma das análises houve violação da esfericidade dos dados. Assim, à ANOVA de medidas repetidas para comparações múltiplas foi empregada. O teste post-hoc de Fisher foi utilizado para localização das diferenças. A significância foi estabelecida em $P \leq 0,05$. Os dados estão apresentados em média \pm desvio-padrão. Todos os cálculos estatísticos foram realizados por meio do software SPSS, versão 17.0.

\section{RESULTADOS}

Sessenta por cento dos sujeitos voluntários da presente investigação eram do sexo feminino. A idade da amostra variou entre 20 e 60 anos, massa corporal entre 48 e 89 kg, estatura entre 1,46 e 1,79 m e índice de massa corporal entre 19 e $31 \mathrm{~kg} / \mathrm{m}^{2}$. Não houve diferença para idade e nos indicadores antropométricos entre os sujeitos do sexo masculino e feminino. Os dados de característica geral da amostra, separados por sexo, são apresentados na Tabela 2.

Figura 1 apresenta os valores referente à percepção subjetiva de esforço durante as sessões de exercício aeróbico contínuo e intervalado. Não foram identificadas diferenças entre as sessões (interação). Porém, em relação aos momentos houve diferença significativa entre todos os momentos, (com exceção do $20^{\circ}$ para $25^{\circ}$ minuto) durante a sessão de exercício contínuo. Já para o exercício intervalado houve menor contundência na elevação da percepção subjetiva de esforço de momento para momento.

Não foram observadas diferenças significativas nos parâmetros hemodinâmicos e autonômicos no momento pré-intervenção entre os grupos experimentais controle, exercício aeróbico contínuo e exercício aeróbico intervalado (Tabela 3).

O comportamento subagudo da pressão arterial sistólica, diastólica e média após a realização das três sessões experimentais são apresentadas na Tabela 3. Aos 60 minutos pós-exercício, a pressão arterial sistólica aumentou na sessão controle e reduziu após a realização de exercício contínuo em relação aos valores de repouso. Além disso, após 60 minutos da sessão de exercício contínuo a pressão arterial sistólica reduziu em relação ao grupo controle. Não houve alterações subagudas na pressão arterial sistólica após a sessão de exercício intervalado.

Em relação às respostas subagudas na pressão arterial diastólica, foram identificadas diferenças significativas em relação ao repouso aos 10 e 40 minutos após a sessão de exercício intervalado. Não houve alteração após a sessão de exercício contínuo e controle na pressão arterial diastólica. A pressão arterial média aumentou significativamente aos 60 minutos em relação aos valores de repouso após a sessão controle. Nas demais sessões experimentais não houve alterações.

A Tabela 4 apresenta os indicadores de atuação autonômica obtidos no repouso e durante 30 e 60 minutos pós-exercício. Considerando as análises do domínio do tempo, a variabilidade global (SDNN) ficou

Tabela 2. Parâmetros hemodinâmicos e autonômicos no momento pré-intervenção (controle, contínuo e intervalado)

\begin{tabular}{|c|c|c|c|c|}
\hline & Controle & Contínuo & Intervalado & $P$ \\
\hline PAS (mmHg) & $115,08 \pm 11,62$ & $115,56 \pm 8,20$ & $111,64 \pm 25,74$ & 0,674 \\
\hline PAD (mmHg) & $75,88 \pm 7,22$ & $76,92 \pm 7,87$ & $72,96 \pm 17,47$ & 0,474 \\
\hline PAM $(\mathrm{mmHg})$ & $88,80 \pm 8,00$ & $89,72 \pm 7,53$ & $85,80 \pm 19,89$ & 0,546 \\
\hline SDNN (ms) & $63,95 \pm 22,50$ & $69,33 \pm 21,12$ & $63,88 \pm 26,74$ & 0,694 \\
\hline RMSSD (ms) & $36,09 \pm 16,35$ & $36,95 \pm 13,99$ & $35,11 \pm 16,39$ & 0,935 \\
\hline pNN50 (\%) & $12,86 \pm 12,39$ & $13,23 \pm 9,68$ & $13,00 \pm 11,30$ & 0,994 \\
\hline$L F_{(n u)}$ & $97,63 \pm 91,10$ & $77,38 \pm 12,06$ & $78,05 \pm 12,73$ & 0,411 \\
\hline$H F_{(n u)}$ & $21,90 \pm 11,80$ & $21,52 \pm 11,92$ & $20,77 \pm 12,72$ & 0,957 \\
\hline LF/HF (ms2) & $4,22 \pm 2,79$ & $4,71 \pm 3,72$ & $4,83 \pm 3,50$ & 0,827 \\
\hline
\end{tabular}

Média \pm desvio-padrão. $\mathrm{PAS}=$ pressão arterial sistólica; $\mathrm{PAD}=$ pressão arterial diastólica; $\mathrm{PAM}=$ pressão arterial média; SDNN= desvio padrão da média de todos os intervalos RR normais; RMSSD= raiz quadrada da média das diferenças sucessivas ao quadrado, entre os intervalos R-R normais adjacentes; pNN50= percentagem de intervalos RR normais adjacentes com diferença de duração maior que 50 milissegundos; $\mathrm{LF}=$ componente de baixa frequência; $\mathrm{HF}=$ componente de alta frequência; $L F / H F=$ razão $L F / H F$. 


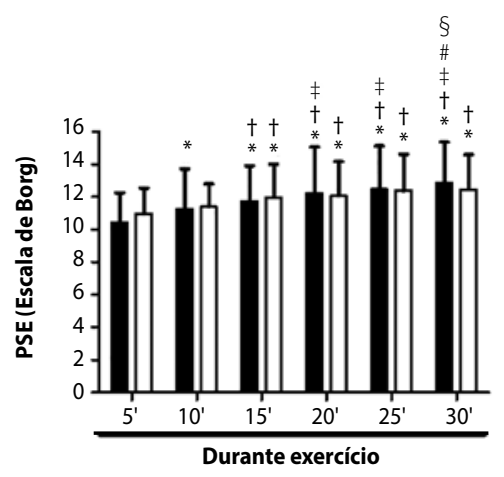

Figura 1. Percepção subjetiva de esforço (PSE) durante a realização das sessões de exercício. Barra escura (preta)= Exercício contínuo; Barra clara (branca)= Exercício intervalado. Média $\pm D P$. ${ }^{*}=P \leq 0,05$ vs $5^{\prime} ; \dagger=P \leq 0,05$ vs $10^{\prime} ; \neq=P \leq 0,05$ vs $15^{\prime} ; \#=P \leq 0,05$ vs $20^{\prime} ; \S=P \leq 0,05$ vs $25^{\prime}$.

Tabela 3. Comportamento subagudo da pressão arterial sistólica, diastólica e média pós-exercício (média \pm desvio-padrão).

\begin{tabular}{c|c|c|c|c|c|c|c}
\hline & Pré & $\mathbf{1 0}$ min & 20min & 30min & 40min & 50min & 60min \\
\hline \multicolumn{8}{c}{ PAS } \\
\hline Controle & $115 \pm 12$ & $115 \pm 10$ & $115 \pm 13$ & $115 \pm 10$ & $117 \pm 10$ & $118 \pm 12$ & $120 \pm 12^{*}$ \\
\hline Contínuo & $115 \pm 8$ & $117 \pm 10$ & $113 \pm 9$ & $114 \pm 9$ & $114 \pm 11$ & $113 \pm 12$ & $112 \pm 11^{* \dagger}$ \\
\hline Intervalado & $112 \pm 26$ & $116 \pm 10$ & $116 \pm 8$ & $114 \pm 11$ & $114 \pm 14$ & $114 \pm 11$ & $115 \pm 11$ \\
\hline
\end{tabular}

\begin{tabular}{c|c|c|c|c|c|c|c}
\hline \multicolumn{1}{c}{ PAD } \\
\hline Controle & $76 \pm 7$ & $75 \pm 8$ & $76 \pm 11$ & $76 \pm 9$ & $76 \pm 9$ & $77 \pm 9$ & $78 \pm 9$ \\
\hline Contínuo & $77 \pm 8$ & $79 \pm 8^{*}$ & $77 \pm 8$ & $77 \pm 9$ & $76 \pm 7$ & $76 \pm 8$ & $77 \pm 9$ \\
\hline Intervalado & $73 \pm 17$ & $79 \pm 8$ & $77 \pm 9$ & $76 \pm 9$ & $79 \pm 11^{*}$ & $76 \pm 8$ & $77 \pm 9$ \\
\hline
\end{tabular}

\begin{tabular}{l|c|c|c|c|c|c|c}
\hline \multicolumn{1}{c}{ PAM } \\
\hline Controle & $89 \pm 8$ & $89 \pm 8$ & $89 \pm 12$ & $89 \pm 8$ & $89 \pm 9$ & $90 \pm 9$ & $91 \pm 9^{*}$
\end{tabular}

\begin{tabular}{c|c|c|c|c|c|c|c} 
Controle & $89 \pm 8$ & $89 \pm 8$ & $89 \pm 12$ & $89 \pm 8$ & $89 \pm 9$ & $90 \pm 9$ & $91 \pm 9^{*}$ \\
\hline Contínuo & $90 \pm 7$ & $90 \pm 8$ & $90 \pm 8$ & $89 \pm 8$ & $88 \pm 8$ & $88 \pm 9$ & $88 \pm 9$ \\
\hline
\end{tabular}

\begin{tabular}{l|c|c|c|c|c|c|c|}
\hline Intervalado & $86 \pm 20$ & $87 \pm 20$ & $86 \pm 19$ & $85 \pm 20$ & $86 \pm 21$ & $85 \pm 19$ & $86 \pm 20$ \\
\hline
\end{tabular}

$\mathrm{PAS}=$ pressão arterial sistólica; $\mathrm{PAD}=$ pressão arterial diastólica; $P$ AM: pressão arterial média. ${ }^{*}=P \leq 0,05$ vs Pré; ${ }^{t}=P \leq 0,05$ vs Controle.

elevada em relação aos valores de repouso e também em relação à sessão de exercício contínuo, para a sessão controle durante os últimos 30 minutos da fase subaguda pós-intervenção.

Os indicadores de variabilidade curta, predominantemente parassimpáticos apresentaram aumento em relação ao momento pré-intervenção após a sessão controle (30 min [RMSSD] e 60 min [RMSSD e pNN50]). Por outro lado, na sessão de exercício esses indicadores (RMSSD e pNN50) se reduziram aos 30 minutos em ambas as sessões (contínuo e intervalado) e foram também menores em relação à sessão controle. Além disso, o indicador pNN50 permaneceu reduzido aos 60 minutos após a sessão de exercício contínuo, tanto em relação ao repousou, quanto para a sessão controle.

Nos indicadores do domínio da frequência houve elevação da variabilidade global, aferida pelo componente de baixa frequência, após a sessão de exercício contínuo, em relação à sessão controle. Após o exercício intervalado, houve elevação do componente de alta frequência (essencialmente parassimpático) somente após a sessão de exercício intervalado em relação aos valores de repouso. Não houve diferenças em relação ao tempo ou entre os grupos na razão LF/HF.

A Tabela 5 apresenta os parâmetros ambulatoriais após as sessões experimentais. Os valores médios da pressão arterial sistólica, pressão arterial diastólica e pressão arterial média foram diferentes em relação aos períodos de vigília, sono e 24 horas para todas as sessões experimentais. Por outro lado, não foram identificadas interações.

\section{DISCUSSÃO}

O presente estudo teve como objetivo analisar o impacto de uma única sessão de exercício contínuo e intervalado sobre as respostas pressóricas subagudas (laboratoriais) e agudas (ambulatórias). Além disso, foram investigadas as respostas subagudas nos indicadores de atuação autonômica. Não é de conhecimento dos autores nenhuma outra investigação que se propôs as avaliar o possível efeito hipotensivo pós-exercício aeróbico intervalado em monitorização de 24 horas de sujeitos normotensos.

A intensidade de exercício estabelecida na presente investigação (60-70\% $\mathrm{FC}_{\text {res }}$ ) seguiu os valores estabelecidos em outras investigações que identificaram ocorrência de hipotensão pós-exercício em amostra normotensa ${ }^{18,19}$. Nesse sentido, percebe-se que não existe diferença na percepção subjetiva de esforço entre as sessões de exercício contínuo e intervalado com duração de atividade de 30 minutos. Por outro lado, na sessão de exercício contínuo existe um aumento intra-série sistemático entre os momentos 5, 10,15, 20 e 30 minutos, indicando que na sessão de exercício contínuo o aumento progressivo da sensação de esforço é mais linear

Vale destacar também que a ocorrência de hipotensão pós-exercício foi identificada somente para a sessão de exercício contínuo,

Tabela 4. Comportamento dos indicadores de atuação autonômica pós-exercício (média \pm desvio-padrão).

\begin{tabular}{|c|c|c|c|}
\hline & Pré & $30 \mathrm{~min}$ & $60 \mathrm{~min}$ \\
\hline \multicolumn{4}{|c|}{ SDNN } \\
\hline Controle & $63,95 \pm 22,50$ & $72,27 \pm 21,94$ & $83,77 \pm 25,30^{*}$ \\
\hline Contínuo & $69,33 \pm 21,12$ & $69,57 \pm 18,34$ & $63,76 \pm 15,96^{\dagger}$ \\
\hline Intervalado & $63,89 \pm 26,74$ & $67,89 \pm 19,66$ & $70,00 \pm 29,48$ \\
\hline \multicolumn{4}{|c|}{ RMSSD } \\
\hline Controle & $36,09 \pm 16,35$ & $41,82 \pm 19,96^{*}$ & $50,55 \pm 31,19^{*}$ \\
\hline Contínuo & $36,95 \pm 13,99$ & $27,05 \pm 12,20^{*+}$ & $35,43 \pm 14,81$ \\
\hline Intervalado & $35,11 \pm 16,39$ & $28,22 \pm 16,04^{* \dagger}$ & $43,39 \pm 29,99$ \\
\hline \multicolumn{4}{|c|}{ pNN50 } \\
\hline Controle & $12,86 \pm 12,39$ & $15,14 \pm 13,25$ & $17,23 \pm 12,37^{*}$ \\
\hline Contínuo & $13,24 \pm 9,68$ & $5,71 \pm 5,72^{*+}$ & $9,71 \pm 7,39^{*+}$ \\
\hline Intervalado & $13,00 \pm 11,30$ & $6,67 \pm 8,64^{* \dagger}$ & $11,17 \pm 10,77$ \\
\hline \multicolumn{4}{|c|}{$\mathrm{LF}_{(\mathrm{nu})}$} \\
\hline Controle & $97,64 \pm 91,10$ & $74,36 \pm 11,62$ & $77,00 \pm 9,52$ \\
\hline Contínuo & $77,38 \pm 12,06$ & $80,76 \pm 8,79^{\dagger}$ & $77,90 \pm 10,86$ \\
\hline Intervalado & $78,06 \pm 12,75$ & $79,17 \pm 8,40$ & $71,78 \pm 16,65$ \\
\hline \multicolumn{4}{|c|}{$\mathrm{HF}_{(\mathrm{nu})}$} \\
\hline Controle & $21,91 \pm 11,80$ & $24,59 \pm 11,54$ & $22,00 \pm 9,53$ \\
\hline Contínuo & $21,52 \pm 11,92$ & $18,19 \pm 8,74$ & $21,05 \pm 10,79$ \\
\hline Intervalado & $20,78 \pm 12,72$ & $19,78 \pm 8,33$ & $27,17 \pm 16,55^{*}$ \\
\hline \multicolumn{4}{|c|}{$\mathrm{LF} / \mathrm{HF}_{(\mathrm{ms})}{ }^{2}$} \\
\hline Controle & $4,23 \pm 2,79$ & $4,71 \pm 3,71$ & $4,83 \pm 3,50$ \\
\hline Contínuo & $3,64 \pm 2,75$ & $4,76 \pm 2,44$ & $4,06 \pm 1,89$ \\
\hline Intervalado & $3,86 \pm 2,83$ & $4,43 \pm 3,01$ & $3,56 \pm 2,99$ \\
\hline
\end{tabular}

SDNN= desvio padrão da média de todos os intervalos RR normais; $R M S S D=$ raiz quadrada da média das diferenças sucessivas ao quadrado, entre os intervalos R-R normais adjacentes; pNN50= percentagem de intervalos RR normais adjacentes com diferença de duração maior que 50 milissegundos; $L_{\text {(nu) }}=$ componente de baixa frequência (unidades normalizadas); $\mathrm{HF}_{(\mathrm{nu})}=$ componente de alta frequência (unidades normalizadas); $\mathrm{LF} / \mathrm{HF}=$ razão $\mathrm{LF} / \mathrm{HF}$. ${ }^{*}=P \leq 0,05$ vs Pré; ${ }^{\dagger}=P \leq 0,05$ vs Controle.

Tabela 5. Parâmetros ambulatoriais após as sessões experimentais.

\begin{tabular}{c|c|c|c}
\hline Variável ambulatorial & Controle & Contínuo & Intervalado \\
\hline Vigilia - PAS & $119,16 \pm 9,15$ & $118,68 \pm 8,41$ & $118,50 \pm 8,59$ \\
\hline Sono - PAS & $106,16 \pm 8,66^{\dagger}$ & $102,44 \pm 7,97^{\dagger}$ & $106,87 \pm 9,12^{\dagger}$ \\
\hline $24 \mathrm{~h}-$ PAS & $116,36 \pm 8,60^{*+}$ & $114,84 \pm 7,95^{*+}$ & $115,95 \pm 8,68^{* \dagger}$ \\
\hline Vigilia - PAD & $77,64 \pm 9,03$ & $76,32 \pm 7,16$ & $76,25 \pm 6,85$ \\
\hline Sono - PAD & $63,96 \pm 7,69^{\dagger}$ & $61,40 \pm 6,37^{\dagger}$ & $63,12 \pm 7,67^{\dagger}$ \\
\hline $24 h-$ PAD & $74,64 \pm 8,36^{* \dagger}$ & $72,80 \pm 6,95^{* \dagger}$ & $73,33 \pm 17,44^{* \dagger}$ \\
\hline Vigilia - PAM & $96,40 \pm 7,94$ & $96,04 \pm 7,58$ & $96,25 \pm 7,33$ \\
\hline Sono - PAM & $83,32 \pm 8,01^{\dagger}$ & $79,88 \pm 7,18^{\dagger}$ & $82,92 \pm 7,88^{\dagger}$ \\
\hline $24 h-$ PAM & $93,60 \pm 7,61^{* \dagger}$ & $92,28 \pm 7,09^{* \dagger}$ & $93,16 \pm 7,43^{* \dagger}$ \\
\hline
\end{tabular}

Média \pm Desvio padrão. $P A S=$ pressão arterial sistólica; $P A D=$ pressão arterial diastólica; $P A M=$ pressão arterial média. ${ }^{*}=P \leq 0,05$ vs Sono; ${ }^{\dagger}=P \leq 0,05$ vs Vigília. 
especificamente aos 60 minutos, sendo identificada em relação ao repouso e a também à sessão controle. Nesse sentido, aparentemente a redução da pressão arterial poderia estar atrelada à sensação subjetiva de esforço, no entanto as correlações entre a variação na percepção subjetiva de esforço e média pressórica pós-exercício foram fracas ( $r=0,127,0,046$ e 0,027 para pressão arterial sistólica, diastólica e média, respectivamente). Por outro lado, em investigação anterior ${ }^{11}$ que acompanhou as respostas pressóricas durante 20 minutos pós-exercício, os autores identificaram resultados contrários aos da presente investigação. Nesse estudo, quando submetidos à sessão de exercício intervalado os sujeitos apresentaram maior magnitude de queda da pressão arterial em relação à sessão de exercício contínuo. Esse efeito divergente pode estar atrelado ao fato de que a amostra do estudo de Jones et al. ${ }^{11}$ tenha sido composta por indivíduos fisicamente ativos, uma vez que as repostas hipotensivas pós-exercício, apesar de aparentemente não serem distintas ${ }^{20}$ podem se manifestar em momentos diferentes devido à provável diferença em relação aos mecanismos hipotensores ${ }^{20}$.

Outro fato interessante observado foi o aumento da pressão arterial sistólica na sessão controle em relação aos valores de repouso pré-intervenção. Comumente utilizada como estratégia controle, a manutenção do sujeito por um longo período de tempo em posição sentada pode gerar ajustes cardiovasculares decorrente do estresse postural e elevar a pressão arteria| ${ }^{21}$. Isso ocorreu independentemente da influência mais acentuada do sistema nervoso parassimpático, identificada pelo aumento nos índices RMSSD e pNN50 sobre o grupo controle. Aparentemente, independente da ação central favorável à estabilidade da pressão arterial, a compressão dos vasos na região do quadril e os ajustes periféricos decorrentes dessa restrição de fluxo podem desencadear ajustes periféricos que elevam a pressão arteria ${ }^{21}$. Nesse sentido, influências sobre o grupo controle podem ser consideradas como relevantes para interpretação de dados oriundos de pesquisas envolvendo monitorização de variáveis cardiovasculares ao longo do tempo.

Considerando as respostas autonômicas subagudas é possível inferir que a redução da pressão arterial pós-exercício tenha sido mais influenciada por fatores periféricos de vasodilatação do que propriamente ajustes centrais oriundos do sistema nervoso central. Obviamente é preciso cuidado na interpretação dos resultados de indicadores autonômicos, uma vez que os dados de variabilidade da frequência cardíaca se referem à atuação autonômica especificamente sobre o coração ${ }^{16}$.

Em relação às repostas ambulatoriais da pressão arterial foi identificado somente diferença em relação aos períodos (vigília, sono e 24h) para todas as condição experimentais. Por outro lado, não foram encontradas diferença entre as sessões (interação) em nenhum dos períodos. Ao estabelecer relações com outros estudos disponíveis na literatura com monitorização ambulatorial após sessões de exercícios especificamente contínuos, uma vez que não foram identificados estudos com exercício intervalado, verifica-se grande variabilidade. Em estudo realizado com mulheres da raça branca não houve resposta significativa da sessão de exercício sobre a pressão arterial ambulatoria|22. Nesse sentido, vale destacar que a amostra do presente estudo foi composta por indivíduos de ambos os sexos e que $60 \%$ da amostra foi composta por mulheres.
Outro estudo ${ }^{23}$ conduzido pelo mesmo grupo de pesquisadores também não identificou a ocorrência de hipotensão pós-exercício em amostra normotensa durante acompanhamento ambulatorial de sete horas durante vigília. Na mesma direção, outras investigações também não encontraram efeito hipotensivo em normotensos de ambos os sexos após exercício contínuo durante monitorização ambulatorial24,25.

Porém, outros estudos têm identificado hipotensão pós-exercício aeróbico contínuo em monitorização ambulatorial da pressão

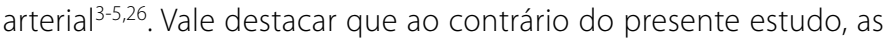
investigações de Fullick et al. ${ }^{3}$ e Jones et al. ${ }^{4}$ foram realizadas com amostra de sujeitos praticantes regulares de exercício aeróbico. Por outro lado, os estudos com amostra considerada sedentária ${ }^{5}$ 6, apresentou respostas divergentes em relação aos achados nesta investigação. Após análise dos métodos de investigação empregados nos referidos estudos, foi identificado que todos submeteram os sujeitos a exercício em cicloergômetro. Inversamente, na presente investigação os sujeitos foram submetidos à sessão de exercício em esteira ergométrica. Atualmente não existe suporte teórico indicando que as respostas hipotensivas agudas podem estar relacionadas ao tipo de ergômetro utilizado, uma vez que outros estudos de acompanhamento subagudo têm identificado a ocorrência de hipotensão pós-exercício em protocolos executados em esteira ergométrica com amostra de normotensos sedentários $27,28$.

Aparentemente, as respostas hipotensivas agudas (24 horas) tanto do exercício contínuo, quanto do intervalado podem ser distintas da presente investigação. Em estudo conduzido por Ciolac et al. ${ }^{29} \mathrm{com}$ amostra de ambos os sexos e protocolo realizado em cicloergômetro, foram identificadas reduções significativas na média de 24 horas para pressão arterial sistólica e média após sessão de exercício contínuo e intervalado. Nesse sentido, é preciso considerar que indivíduos hipertensos tendem a apresentar maior magnitude de hipotensão pós-exercício, estando esse fenômeno de acordo com a teoria dos valores iniciais ${ }^{30}$.

Os resultados apresentados na presente investigação devem ser interpretados considerando que as avaliações, apesar de sempre executadas no período da tarde, não foram realizadas exatamente no mesmo horário. Além disso, os sujeitos não possuíam o mesmo hábito de horários e tempo despendido em sono e vigília, o que impediu a análise das respostas pressóricas pós-exercício momento a momento.

\section{CONCLUSÃO}

Com base nos resultados é possível concluir que uma sessão de exercício aeróbico contínuo causa redução subaguda da pressão arterial em adultos normotensos. Sessão única de exercício aeróbico contínuo e intervalado não promove redução da pressão arterial ambulatorial na média dos períodos de sono e vigília. Os indicadores autonômicos do domínio do tempo relacionados à atuação parassimpática permanecem inferiores até 30 minutos pós-exercício.

Todos os autores declararam não haver qualquer potencial conflito de interesses referente a este artigo.

CONTRIBUIÇÕES DOS AUTORES: Cada autor contribuiu individual e significativamente para o desenvolvimento do manuscrito. JC (0000-0001-5397-5694)* foi o proponente da ideia e realizou a redação do manuscrito. VD (0000-0002-0275-0185)* aplicou o modelo experimental, reuniu os dados clínicos e contribuiu significativamente na redação. DGDC (0000-0001-9917-9992)* realizou a revisão do manuscrito e contribuiu com o conceito intelectual do estudo. Todos os autores participaram ativamente da discussão dos resultados, revisão e aprovação final. *ORCID (Open Researcher and Contributor ID).

\section{REFERÊNCIAS}

1. Kenney MJ, Seals DR. Postexercise hypotension. Key features, mechanisms, and clinical significance. Hypertension. 1993;22(5):653-64

2. Hamer M. The anti-hypertensive effects of exercise: integrating acute and chronic mechanisms. Sports Med. 2006;36(2):109-16.
3. Fullick S, Morris $\mathrm{C}$, Jones $\mathrm{H}$, Atkinson G. Prior exercise lowers blood pressure during simulated night-work with different meal schedules. Am J Hypertens. 2009;22(8):835-41.

4. Jones $\mathrm{H}$, George K, Edwards B, Atkinson G. Exercise intensity and blood pressure during sleep. Int J Sports Med. 2009;30(2):94-9. 
5. Blanchard BE, Tsongalis GJ, Guidry MA, LaBelle LA, Poulin M, Taylor AL, et al. RAAS polymorphisms alter the acute blood pressure response to aerobic exercise among men with hypertension. Eur J Appl Physiol. 2006;97(1):26-33.

6. Bermudes AM, Vassallo DV, Vasquez EC, Lima EG. Ambulatory blood pressure monitoring in normotensive individuals undergoing two single exercise sessions: resistive exercise training and aerobic exercise training. Arq Bras Cardiol. 2004;82(1):65-71.

7. Prista A, Macucule CF, Queiroz AC, Silva ND, Jr., Cardoso CG, Jr., TinucciT, et al. A bout of resistance exercise following the 2007 AHA guidelines decreases asleep blood pressure in Mozambican men. J Strength Cond Res. 2013;27(3):786-92.

8. Queiroz AC, Kanegusuku H, Chehuen MR, Costa LA, Wallerstein LF, Dias da Silva VJ, et al. Cardiac work remains high after strength exercise in elderly. Int J Sports Med. 2013;34(5):391-7.

9. Queiroz AC, Sousa JC, Cavalli AA, Silva ND, Jr., Costa LA, Tobaldini E, et al. Post-resistance exercise hemodynamic and autonomic responses: Comparison between normotensive and hypertensive men Scand J Med Sci Sports. 2015;25(4):486-94.

10. Pescatello LS, Franklin BA, Fagard R, FarquharWB, Kelley GA, Ray CA. American College of Sports Medicine position stand. Exercise and hypertension. Med Sci Sports Exerc. 2004;36(3):533-53.

11. Jones $H$, Taylor CE, Lewis NC, George $K$, Atkinson G. Post-exercise blood pressure reduction is greate following intermittent than continuous exercise and is influenced less by diurnal variation. Chronobiol Int. 2009;26(2):293-306

12. Gordon CC, Chumlea WC, Roche AF. Stature, recumbent length, and weight. Lohman TG, Roche AF, Martorell R, editors. Champaign: Human Kinetics Books;1988. 3-8 p

13. Coleman A, Freeman P, Steel S, Shennan A. Validation of the Omron MX3 Plus oscillometric blood pressure monitoring device according to the European Society of Hypertension international protocol. Blood Press Monit. 2005:10(3):165-8.

14. Pickering TG, Hall JE, Appel $\sqcup$, Falkner BE, Graves J, Hill MN, et al. Recommendations for blood pressure measurement in humans and experimental animals: Part 1: blood pressure measurement in humans: a statement for professionals from the Subcommittee of Professional and Public Education of the American Heart Association Council on High Blood Pressure Research. Hypertension. 2005;45(1):142-61.

15. Quintana DS, Heathers JA, Kemp AH. On the validity of using the Polar RS800 heart rate monitor for heart rate variability research. Eur J Appl Physiol. 2012(Epub ahead of print).

16. Heart rate variability: standards of measurement, physiological interpretation and clinical use. Task Force of the European Society of Cardiology and the North American Society of Pacing and Electrophysiology.
Circulation. 1996;93(5):1043-65.

17. Borg G. Borg's Perceived Exertion and Pain Scales. Champaign: Human Kinetics;1998

18. Ruiz RJ, Simão R, Saccomani MG, Casonatto J, Alexander JL, Rhea M, et al. Isolated and combined effects of aerobic and strength exercise on post-exercise blood pressure and cardiac vagal reactivation in normotensive men. J Strength Cond Res. 2011;25(3):640-5.

19. Endo MY, Shimada K, Miura A, Fukuba Y. Peripheral and central vascular conductance influence on post-exercise hypotension. J Physiol Anthropol. 2012;31(32):1-7.

20. Senitko AN, Charkoudian N, Halliwill JR. Influence of endurance exercise training status and gender on postexercise hypotension. J Appl Physiol. 2002;92(6):2368-74.

21. Gotshall RW, Aten LA, Yumikura S. Difference in the cardiovascular response to prolonged sitting in men and women. Can J Appl Physiol. 1994;19(2):215-25.

22. Pescatello LS, Bairos L, Vanheest JL, Maresh CM, Rodriguez NR, Moyna NM, et al. Postexercise hypotension differs between white and black women. Am Heart J. 2003:145(2):364-70.

23. Pescatello LS, Miller B, Danias PG, Werner M, Hess M, Baker C, et al. Dynamic exercise normalizes resting blood pressure in mildly hypertensive premenopausal women. Am Heart J. 1999;138(5 Pt 1):916-21.

24. Wallace JP, Bogle PG, King BA, Krasnoff JB, Jastremski CA. The magnitude and duration of ambulatory blood pressure reduction following acute exercise. J Hum Hypertens. 1999;13(6):361-6.

25. Brownley KA, West SG, Hinderliter AL, Light KC. Acute aerobic exercise reduces ambulatory blood pressure in borderline hypertensive men and women. Am J Hypertens. 1996;9(3):200-6.

26. Forjaz CL, Tinucci T, Ortega KC, Santaella DF, Mion D, Jr., Negrao CE. Factors affecting post-exercise hypotension in normotensive and hypertensive humans. Blood Press Monit. 2000;5(5-6):255-62.

27. Harvey PJ, Morris BL, Kubo T, Picton PE, Su WS, Notarius CF, et al. Hemodynamic after-effects of acute dynamic exercise in sedentary normotensive postmenopausal women.J Hypertens. 2005;23(2):285-92.

28. Headley SA, Claiborne JM, Lottes CR, Korba CG. Hemodynamic responses associated with post-exercise hypotension in normotensive black males. Ethn Dis. 1996;6(1-2):190-201.

29. Ciolac EG, Guimaraes GV, VM DA, Bortolotto LA, Doria EL, Bocchi EA. Acute effects of continuous and interval aerobic exercise on 24-h ambulatory blood pressure in long-term treated hypertensive patients. Int J Cardiol. 2008;133(3):381-7.

30. Wilder J. The law of initial value in neurology and psychiatry;facts and problems. J Nerv Ment Dis 1957;125(1):73-86. 